\title{
Structural heterogeneity of the mammalian polycomb repressor complex in immune regulation
}

\author{
Seok-Jin Kang ${ }^{1}$ and Taehoon Chun (1)
}

\begin{abstract}
Epigenetic regulation is mainly mediated by enzymes that can modify the structure of chromatin by altering the structure of DNA or histones. Proteins involved in epigenetic processes have been identified to study the detailed molecular mechanisms involved in the regulation of specific mRNA expression. Evolutionarily well-conserved polycomb group ( $\mathrm{PcG}$ ) proteins can function as transcriptional repressors by the trimethylation of histone $\mathrm{H} 3$ at the lysine 27 residue (H3K27me3) and the monoubiquitination of histone H2A at the lysine 119 residue (H2AK1 19ub). PcG proteins form two functionally distinct protein complexes: polycomb repressor complex 1 (PRC1) and PRC2. In mammals, the structural heterogeneity of each PRC complex is dramatically increased by several paralogs of its subunit proteins. Genetic studies with transgenic mice along with RNA-seq and chromatin immunoprecipitation (ChIP)-seq analyses might be helpful for defining the cell-specific functions of paralogs of PCG proteins. Here, we summarize current knowledge about the immune regulatory role of PCG proteins related to the compositional diversity of each PRC complex and introduce therapeutic drugs that target PcG proteins in hematopoietic malignancy.
\end{abstract}

\section{Introduction}

In eukaryotes, the alteration of chromatin structure is one of the main methods for modifying cell phenotypes by regulating specific DNA replication and mRNA transcription ${ }^{1}$. In addition to DNA methylation, changing the properties of certain amino acid residues at histones is a major method for modifying the structure of chromatin. The enzymes involved in the acetylation, methylation, ubiquitination, and phosphorylation of histones have been identified and extensively studied to define the biological function of each enzyme ${ }^{2}$. Many studies have provided evidence that histone modification plays a decisive role in cell fates such as carcinogenesis, differentiation, proliferation, and senescence ${ }^{3}$.

Polycomb group (PcG) proteins were originally identified from fruit flies. They are well conserved from invertebrates to mammals during evolution. PcG proteins can

\footnotetext{
Correspondence: Taehoon Chun (tchun@korea.ac.kr)

'Department of Biotechnology, College of Life Sciences and Biotechnology, Korea University, Seoul 02841, Republic of Korea
}

act as transcriptional repressors by inhibiting the mRNA transcription of specific gene loci through the trimethylation or monoubiquitination of histones $\mathrm{H} 3$ and $\mathrm{H}_{2} \mathrm{~A}^{4}$. To initiate and maintain such chromatin modification, two distinct protein complexes, polycomb repressor complex 1 (PRC1) and PRC2, work in coordination with each other. PRC2 exhibits methyltransferase activity to add methyl functional groups to specific amino acid residues of histone $\mathrm{H} 3$, while PRC1 exhibits E3 ubiquitin-ligase activity to modify the structure of histone $\mathrm{H} 2 \mathrm{~A}^{4,5}$. Mammalian PRC complexes display structural plasticity because the existence of several paralogs of PcG subunit proteins ${ }^{6}$. In particular, more than 100 different types of mammalian PRC1 complexes may exist based on a simple combinatorial algorithm ${ }^{7}$.

Although recent progress in biochemical and molecular analyses involving transgenic animal techniques has revealed the functional importance of the core subunit of the PcG proteins that regulate mRNA expression through histone modifications, how each of the paralogs of PcG subunit 
A
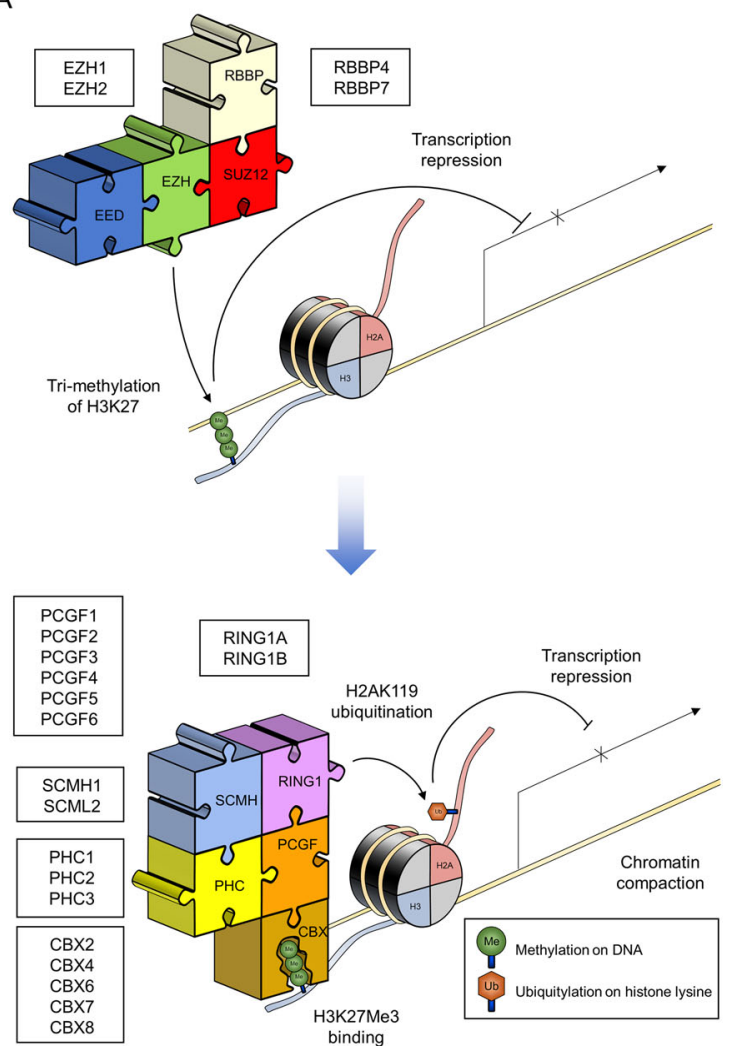

B

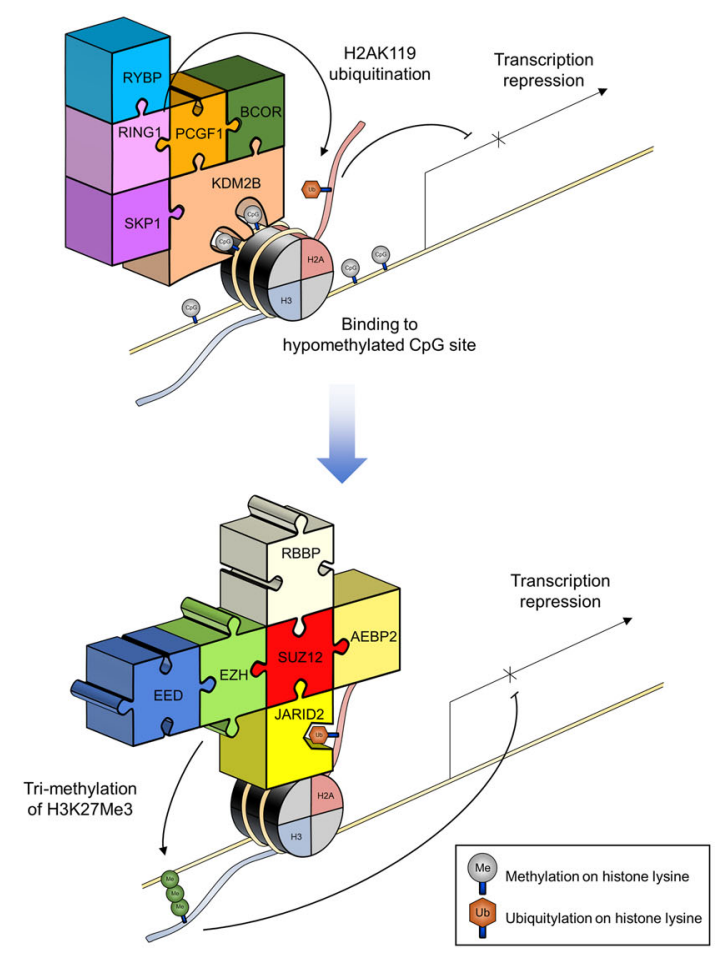

Fig. 1 The repressive mechanism of specific mRNA transcription by PcG proteins through the modification of chromatin structure. Schematic representation of transcriptional repression by PcG proteins according to the 'hierarchical repressive model' (a) and the 'reversehierarchical repressive model' (b). a Core subunits of PRC2 (EED, EZH, SUZ12, RBBP) recognize and repress a target locus by introducing H3K27me3. The CBX subunit of canonical PRC1 (PRC1.2 and PRC1.4) then recognizes the H3K27me3 tag, and canonical PRC1 further represses the target locus by introducing H2AK119. b The KDM2B subunit of noncanonical PRC1 (PRC1.1) recognizes CpG, and PRC1.1 represses the target locus by introducing H2AK119. The JARID2 subunit of PRC2.2 then recognizes the H2AK119 tag, and PRC2.2 further represses the target locus by introducing H3K27me3.

protein interact with each other to orchestrate the fine tuning of chromatin structure remains elusive. In this review, we summarize current knowledge about the immune regulatory role of PcG proteins related to the compositional diversity of each PRC complex. We also introduce therapeutic drugs that target PcG proteins.

\section{Structural heterogeneity related to the function of PcG proteins}

PcG genes were initially identified as genes involved in the regulation of homeotic gene expression, critical for the body axis plan and segment development in fruit flies ${ }^{8}$. PcG proteins are present in plants, nematodes, and metazoan species from flies to mammals, indicating that these proteins are well-conserved transcriptional repressors via the modification of chromatin structure during evolution ${ }^{9}$. Each PcG protein is a subunit of multiprotein complexes categorized by two different functional groups: PRC1 and PRC2 ${ }^{10}$.

Embryonic ectoderm development (EED), suppressor of zeste (SUZ)12, and enhancer of zeste homolog (EZH) are the catalytic core subunits of PRC2. Since EZH has two paralogs (EZH1 and EZH2), two structural variants are found in the catalytic core of PRC2 (Fig. 1a) ${ }^{11}$. EZH2 is the enzymatic subunit of the PRC2 complex, which acts as an S-adenosyl-L-methionine (SAM)-dependent histone methyltransferase via the mono-, di-, or trimethylation of lysine 27 residue at histone $\mathrm{H} 3$ (H3K27me1, H3K27me2, or H3K27me3) (Fig. 1 and Table 1) ${ }^{11-16}$. EZH1 also acts as a methyltransferase with reduced enzyme activity compared to $\mathrm{EZH} 2^{10}$. The SET domain of EZH1 or EZH2, which contains the catalytic core and SAM-binding site, is indispensable for their methyltransferase activity. However, purified EZH1 or EZH2 monomers alone are unable to efficiently exert enzyme activity in vitro because they must bind with two other noncatalytic subunit proteins, SUZ12 and EED (Fig. 1, Tables 1 and 2) 9,11,12,17-21 $^{\text {. SUZ12 }}$ contains a zinc-finger domain that can bind to DNA or RNA and facilitate protein-protein interactions ${ }^{22}$. EED contains WD40 repeats that can putatively bind to H3K27me3 (Table 2) ${ }^{23}$. The fourth member of the PRC2 
Table 1 Each subunit of canonical and noncanonical polycomb complexes in mammals.

\begin{tabular}{llll}
\hline PRC & Core subunits & Classification & Other subunits \\
\hline PRC2.1 & EZH, EED, SUZ12, RBBP & N.D. & PCL, EPOP (C17orf96), LCOR (C10orf12) \\
PRC2.2 & EZH, EED, SUZ12, RBBP & N.D. & AEBP2, JARID2 \\
PRC1.1 & PCGF1 (RNF68, NSPC1), RING1 & Noncanonical & RYBP, KDM2B (FBXL10), BCOR, SKP1 \\
& PCGF1 (RNF68, NSPC1), RING1 & Noncanonical & YAF2, KDM2B (FBXL10), BCOR, SKP1 \\
& PCGF1 (RNF68, NSPC1), RING1 & Noncanonical & CBX8, KDM2B (FBXL10), BCOR, SKP1 \\
PRC1.2 & PCGF2 (MEL-18), RING1 & Canonical & CBX, PHC, SCMH \\
PRC1.3 & PCGF3 (RNF3), RING1 & Noncanonical & RYBP, AUTS2, FBRS, CKII (CSNK2A1) \\
& PCGF3 (RNF3), RING1 & Noncanonical & YAF2, AUTS2, FBRS, CKII (CSNK2A1) \\
PRC1.4 & PCGF4 (BMI-1), RING1 & Canonical & CBX, PHC, SCMH \\
PRC1.5 & PCGF5 (RNF159), RING1 & Noncanonical & RYBP, AUTS2, FBRS, CKII (CSNK2A1) \\
& PCGF5 (RNF159), RING1 & Noncanonical & YAF2, AUTS2, FBRS, CKII (CSNK2A1) \\
PRC1.6 & PCGF6 (RNF134, MBLR), RING1 & Noncanonical & RYBP, WDR5, L3MBTL2, ESF6-TDP1, MGA-MAX, CBX3 (HP1Y) \\
& PCGF6 (RNF134, MBLR), RING1 & Noncanonical & YAF2, WDR5, L3MBTL2, ESF6-TDP1, MGA-MAX, CBX3 (HP1Y) \\
\hline
\end{tabular}

N.D. not determined.

Table 2 Paralogs of each subunit of PRC2 and canonical PRC1 in mammals.

\begin{tabular}{|c|c|c|c|c|c|}
\hline $\begin{array}{l}\text { Core subunit of } \\
\text { PRC2 }\end{array}$ & $\begin{array}{l}\text { Homologs in fruit } \\
\text { flies }\end{array}$ & $\begin{array}{l}\text { Paralogs in } \\
\text { mammals }\end{array}$ & Protein domain & Function & References \\
\hline EED & ESC & none & WD40 repeat & Binding to $\mathrm{H} 3 \mathrm{~K} 27 \mathrm{me} 3$ & 9 \\
\hline \multirow[t]{2}{*}{$\mathrm{EZH}$} & \multirow[t]{2}{*}{$E(Z)$} & $\mathrm{EZH} 1$ & \multirow[t]{2}{*}{ SET domain } & \multirow[t]{2}{*}{ H3K27 methyltransferase } & \multirow[t]{2}{*}{17} \\
\hline & & $\mathrm{EZH} 2$ & & & \\
\hline SUZ12 & $\mathrm{SU}(Z) 12$ & none & Zinc-finger domain & $\begin{array}{l}\text { DNA/RNA binding and protein-protein } \\
\text { interaction }\end{array}$ & 17,18 \\
\hline \multirow[t]{2}{*}{ RBBP } & \multirow[t]{2}{*}{ NURF55 } & RBBP4 & \multirow[t]{2}{*}{ WD40 repeat } & \multirow[t]{2}{*}{ Binding to unmodified nucleosomes } & \multirow[t]{2}{*}{18,19} \\
\hline & & RBBP7 & & & \\
\hline $\begin{array}{l}\text { Core subunit of } \\
\text { canonical PRC1 }\end{array}$ & Fruit fly & Mammalian & Protein domain & Function & References \\
\hline \multirow[t]{2}{*}{ RING1 } & \multirow[t]{2}{*}{ RING } & RING1A (RING1) & \multirow{2}{*}{$\begin{array}{l}\text { RING finger } \\
\text { domain }\end{array}$} & \multirow{2}{*}{$\begin{array}{l}\text { Nucleosome binding and H2AK119 mono- } \\
\text { ubiquitin ligase }\end{array}$} & \multirow[t]{2}{*}{20} \\
\hline & & RING1B (RNF2) & & & \\
\hline \multirow[t]{6}{*}{ PCGF } & \multirow[t]{6}{*}{ PSC } & PCGF1 & \multirow{6}{*}{$\begin{array}{l}\text { RING finger } \\
\text { domain and } \\
\text { RAWUL domain }\end{array}$} & \multirow{6}{*}{$\begin{array}{l}\text { H2AK119 mono-ubiquitin ligase and } \\
\text { protein-protein interaction }\end{array}$} & \multirow[t]{6}{*}{21} \\
\hline & & MEL18 (PCGF2) & & & \\
\hline & & PCGF3 & & & \\
\hline & & BMI1 (PCGF4) & & & \\
\hline & & PCGF5 & & & \\
\hline & & PCGF6 & & & \\
\hline
\end{tabular}

core subunit is retinoblastoma-binding protein 4 (RBBP4) (NURF55) or RBBP7 (Fig. 1, Tables 1 and 2) Whether RBBP $4 / 7$ is included in the catalytic core of PRC2 is still controversial because RBBP 4/7 activity is not required for the catalytic activity of PRC2 in vitro ${ }^{26}$. However, RBBP 4/7 also contains WD40 domains that can bind to histones and facilitate the catalytic activity of PRC2 in vivo ${ }^{26}$. 
In addition to the core subunits of PRC2, several other proteins can bind to these subunits and modulate the enzyme activity of PRC2. Two different types of PRC2 complexes (PRC 2.1 and PRC 2.2) have been identified based on their noncore subunit proteins in humans (Table $1)^{11,12,27}$. PRC2.1 contains three other subunits, including polycomb-like protein (PCL), PRC2-associated LCOR isoform (PALI), elongin $\mathrm{B} / \mathrm{C}$ and $\mathrm{PRC} 2$-associated protein (EPOP) (Table 1) $)^{11,12,28-30}$. PCL has three paralogs: PCL1, PCL2, and PCL3. They are also known as PHF1 (PCL1), MTF2 (PCL2), and PHF19 (PCL3), respectively. PALI, also known as C10ORF12, has two paralogs: PALI1 and PALI2 ${ }^{28-30}$. Three noncore subunit proteins (PCL, PALI and EPOP) can act as enhancers to facilitate the catalytic activity of PRC 2.1. The function of PCL is essential for H3K27me3 by PRC 2.1 because the recognition of H3K36me2/3 by the TUDOR domain of PCL is a prerequisite for PRC 2.1 to introduce $\mathrm{H} 3 \mathrm{~K} 27 \mathrm{me} 3$ marks $^{31}$. PCL is also required for the recognition of unmethylated CpG islands of DNA by PRC $2.1^{32,33}$. PALI1 can facilitate the catalytic activity of PRC2 both in vitro and in vivo ${ }^{34}$. Similar to the phenotype of EZH2-deficient mice, PALI1deficient mice exhibit embryonic lethality ${ }^{34}$. EPOP can mediate the interaction between PRC2.1 and elongin $\mathrm{B} / \mathrm{C}$, which is important for maintaining the transcriptional repression of PRC2's target locus ${ }^{35}$.

Adipocyte enhancer-binding protein 2 (AEBP2) and Jumonji AT-rich interactive domain 2 (JARID2) are additional subunits that for PRC 2.2 along with the PRC2 core subunits (Fig. 1 and Table 1) ${ }^{11,12,27,36}$. Both AEBP2 and JARID2 are required to recruit PRC 2.2 to chromatin by specifically binding to the CpG-rich region of $\mathrm{DNA}^{36}$. Recent studies have indicated that Jarid2-containing PRC 2.2 can specifically recognize and bind to the monoubiquitinated lysine 119 residue at histone H2A (H2AK119 $\mathrm{Ub}$ ) tagged by the PRC1.1 (noncanonical PRC 1) complex (Fig. 1b) ${ }^{37}$. The binding of H2AK119Ub by Jarid2 can further facilitate the methyltransferase activity of PRC 2.2 (Fig. 1b) $)^{37}$.

The subunits of PRC1 complexes are much more diverse

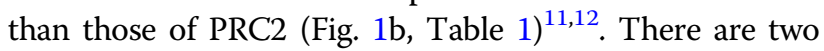
groups of PRC1 complexes categorized based on the original findings in fruit flies. Canonical PRC1 complexes are composed of subunit proteins conserved from flies to mammals, whereas the subunit proteins of noncanonical PRC1 complexes are less conserved in flies ${ }^{38}$. Really interesting new gene 1 (RING) and polycomb group ring finger (PCGF) have been found in both canonical and noncanonical PRC1 complexes, suggesting that these proteins are structurally and functionally essential components $^{38}$. RING proteins exhibit two paralogs (RING1A and RING1B) that possess E3 ubiquitin ligase activity when they are combined with PCGF proteins (H2AK119Ub

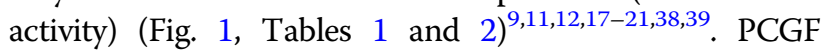

proteins exhibit six paralogs (PCGF1-PCGF6) ${ }^{20}$. Upon interaction with RING proteins, PCGF proteins can increase ubiquitin ligase activity by acting as cofactors ${ }^{39,40}$. Each PCGF paralog (PCGF1 through PCGF6) can be a subunit of different types of PRC1 complexes (PRC1.1

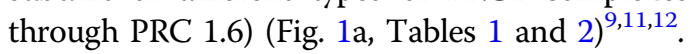

Among the six different types of PRC1 complexes (PRC1.1-PRC 1.6), the PCGF2 (MEL-18)-containing PRC1.2 and PCGF4 (BMI-1)-containing PRC1.4 complexes are classified as canonical PRC1 complexes ${ }^{41}$. Chromobox homologs (CBX) can form canonical PRC1 complexes with RING proteins and PCGF2 (MEL-18) or PCGF4 (BMI-1) (Fig. 1a) ${ }^{41}$. Five CBX paralogs (CBX2, CBX4, CBX6, CBX7, CBX8) have been found to act as subunits of the canonical PRC1 complex in mammals (Fig. 1a) ${ }^{41}$. The proposed role of CBX in the canonical PRC1 complex is to recruit PRC1 to $\mathrm{H} 3 \mathrm{~K} 27 \mathrm{me} 3$ tags because CBX proteins contain chromodomains that recognize the H3K27me3 tag introduced by PRC2 (Fig. 1a) ${ }^{42,43}$. Additionally, polyhomeotic homolog (PHC) and sex comb on midleg homolog (SCMH) can interact with core proteins (RING and PCGF) to form canonical PRC1 complexes (Fig. 1a, Table 1) (4,,45 $^{3}$ PHC proteins exhibit three paralogs (PHC1-PHC3) $)^{3,7}$. SCMH proteins exhibit two paralogs $(\mathrm{SCMH} 1 \text { and } \mathrm{SCMH} 2)^{46}$. Both types of proteins contain a sterile $\alpha$ motif domain that allows them to bind to other canonical PRC1 complex proteins and participate in the recruitment of PRC1 to chromatin (Table 1$)^{44,45}$. PHC proteins also have zincfinger domains that facilitate nucleic acid binding and chromatin compaction ${ }^{46}$.

The noncanonical PRC1 complex is composed of more protein subunits (Table 1$)^{11,12}$. In the noncanonical PRC1 complex, the core subunits (RING1 and PCGF) can interact with ring and YY1 binding protein (RYBP) or YY1-associated factor 2 (YAF2) or CBX8 (Table 1) via $C$ terminal ring finger and WD40 ubiquitin-like (RAWUL) domains ${ }^{12,47}$. Previous observations have indicated that RYBP can compete with CBX for the binding site of RING1B $^{48}$. YAF2 and RYBP occur in the noncanonical PRC1 complex in a mutually exclusive manner, since YAF2 is a homolog of RYBP (Table 1).

The function of the noncanonical PRC1 complex is clearly different from that of the canonical PRC1 complex (Fig. 1). According to the 'hierarchical repressive model', PRC2 can repress a target locus via an H3K27me3 tag. The canonical PRC1 complex can recognize this methylation tag through CBX and further repress a target locus by introducing a H2AK119 mark (Fig. 1a) ${ }^{43}$. Recently, the RYBP-containing noncanonical PRC1 complex has been found to show higher E3 ligase activity than PCGF4RING1B containing canonical PRC1 complex ${ }^{49}$. This finding suggests that another pathway for transcriptional repression exists in addition to the 'hierarchical repressive model'. Indeed, the CxxC DNA-binding domain of 
KDM2B in the PRC1.1 complex can specifically recognize CpG DNA sequences and recruit PRC1.1 to a target locus $^{50,51}$. PRC1.1 then suppresses specific mRNA transcription via an H2AK119ub tag (Fig. 1b) ${ }^{50,51}$. Thereafter, PRC 2.2-containing Jarid2 can specifically recognize and bind the H2AK119Ub tag and further modify the structure of chromatin by introducing an H3K27me3 tag (Fig. $1 \mathrm{~b})^{37,50,51}$. This model is known as the 'reverse hierarchical repressive model' because PRC1.1 first represses the specific transcription of mRNA instead of PRC2.2.

In fruit flies, putative DNA regions recognized by PRCs have been identified, validated, and designated as PcG/ trithorax-group response elements (PREs) ${ }^{2-7}$. The existence of vertebrate PRE sites around CpG-rich sequences has also been suggested ${ }^{36,52}$. However, the conserved DNA-binding motif of mammalian PRCs and the detailed mechanism by which mammalian PRCs recognize specific DNA regions remain elusive. In fruit flies, it has been suggested that the pleiohomeotic (Pho) protein can recognize PREs and guide the core subunits of PRC1 and PRC2 to PREs since the core subunits of PRC2 or PRC1 do not directly bind to $\mathrm{DNA}^{53}$. In vertebrates, YinYang1 (YY1), a Pho homolog, can bind to a conserved DNA region and interact with PRC1 subunits ${ }^{54}$. Therefore, YY1 may recognize PRE sites and guide noncanonical PRC1 by interacting with RYBP or YAF $2^{55}$.

\section{The role of PcG proteins in immune regulation}

A knockout (KO) mouse model and the cell typespecific deletion of PcG genes generated in a conditional knockout (cKO) mouse model using the cre-lox system have been used in most studies to study the function of PcG proteins in immune regulation (Table 3$)^{56-82}$. Except gene encoding RBBP, mice deficient in the genes encoding each core subunit of PRC2 have been generated and characterized (Table 3$)^{56-82}$. Based on animal studies, Ezh1 can partially replace the function of EZh2 in specific cell types ${ }^{83,84}$. For example, Ezh2 is not required for the self-renewal activity of long-term hematopoietic stem cells (LT-HSCs) in adult bone marrow ${ }^{64}$. However, Ezh1deficient mice exhibit immunodeficiency due to a significant loss of the self-renewal activity of $\mathrm{HSCs}^{56}$. Because the INK4a/Arf locus, encoding p16INK4a and p19Arf, which can suppress cell cycle progression, is a target of PcG-mediated repression, the deficiency of certain core subunits of PRC2 and canonical PRC1 can cause the loss of self-renewal activity of $\mathrm{HSCs}^{85}$. In addition to EZH1 deficiency, insufficiency of other subunits of PRC2, including EED or SUZ12, can lead to the loss of the selfrenewal activity of $\mathrm{HSCs}^{64,66}$. The deficiency of some canonical subunits of PRC1, including BMI-1 and PHC1, can also cause the loss of self-renewal activity of $\mathrm{HSCs}^{67,68,75}$. However, other canonical subunits of PRC1, including MEL18, CBX2, CBX8, and $\mathrm{PHC} 2$, do not influence the self-renewal activity of $\mathrm{HSCs}^{72,76,77,79}$. These phenotypic variations observed in each of the mice deficient in different PcG subunits reflect structural heterogeneity depending on the specific stage of cells or tissues due to the redundancy or paralogs of each PcG subunit (Table 3). Cell type-specific roles of various PRC1 and PRC2 complexes have already suggested (Fig. 2) ${ }^{12}$. In support of these ideas, EZH2 expression in LT-HSCs peaks on embryonic day 14.5 and gradually decreases thereafter until 10 months postnatal ${ }^{64}$. However, EZH1 expression in LT-HSCs gradually increases from embryonic day 14.5 to 10 months after birth ${ }^{64}$. BMI-1 and MEL18 expression patterns also follow the paradigm of EZH1/2 expression. BMI-1 is mainly expressed in specific lineage precursors of immune cells, whereas the expression of MEL-18 is correlated with mature immune cell populations ${ }^{86}$. In addition to contributing to the selfrenewal activity of HSCs, PcG proteins participate in the differentiation of hematopoietic progenitor cells (HPCs) into specific lineages of immune cells. The contributions of PRC2 and canonical PRC1 to immune cell differentiation according to the 'hierarchical repressive model' are summarized in Table 3 and Fig. 2.

Studies on the importance of PcG proteins in immune cell function are much less common than studies on the influence of PcG proteins during the differentiation of immune cells (Table 3). Most studies on the functional contribution of PcG proteins to immune cell function have focused on $\mathrm{T}$ cell function (Table 3). The $\mathrm{CD}^{+} \mathrm{T}$ cell-specific deletion of $E z h 2$ or Eed using the CD4-Cre or granzyme B-Cre system revealed that the antigen-specific activation of $\mathrm{CD} 8^{+} \mathrm{T}$ cells requires the function of the PRC2 complex (Table 3$)^{61}$. Interestingly, the contribution of $\mathrm{PcG}$ proteins to $\mathrm{CD} 4^{+} \mathrm{T}$ cell function is controversial because the phenotypes of each of the PcG protein-deficient mice are quite different from each other. For example, $\mathrm{CD} 4^{+} \mathrm{T}$ cell-specific Ezh2 deletion has led to type 2 helper $\mathrm{T}$ cell $\left(\mathrm{Th}_{2}\right)$-prone immunity via the accumulation of memory $\mathrm{Th}_{2}$ cells, which exacerbates allergic diseases (Table 3) ${ }^{59}$. However, Bmi1 and Mel18 knockout mice are defective in $\mathrm{Th}_{2}$ cell differentiation ${ }^{71,73}$. Furthermore, Bmil knockout mice exhibit the enhanced apoptosis of memory $\mathrm{Th}_{2}$ cells ${ }^{69}$.

\section{Current RNA-seq and chromatin immunoprecipitation (ChIP)-seq data for identifying the target loci of PcG proteins}

The phenotypic analysis of transgenic mice in combination with RNA-seq and chromatin immunoprecipitation (ChIP)-seq analyses might be a good approach for identifying additional target loci of PcG proteins or the unique functions of each PcG protein paralog in specific immune cell types. Table 4 summarizes current gene chip and RNA-seq databases generated from specific cell types of transgenic mice or specific cell lines subjected to 


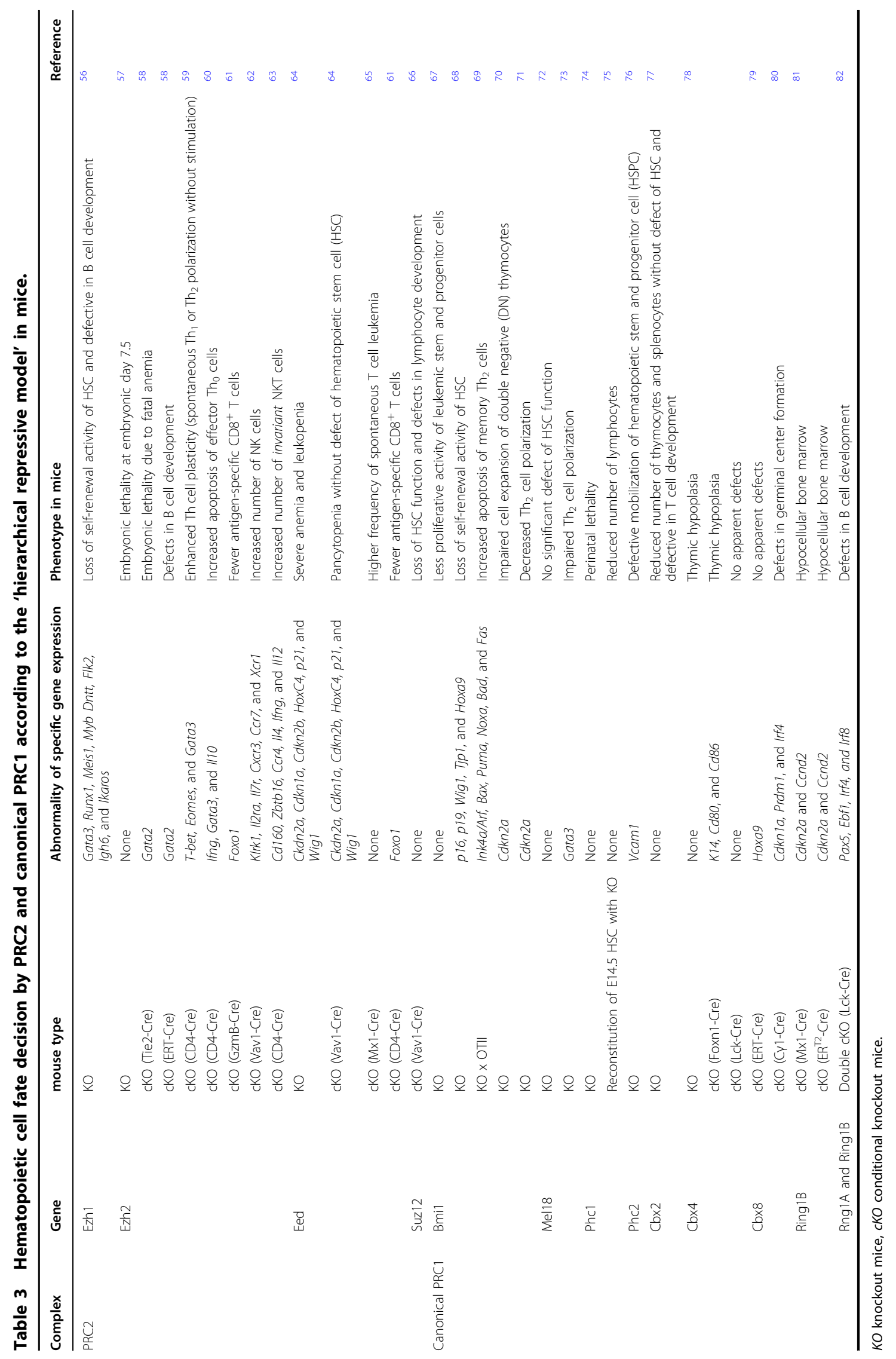




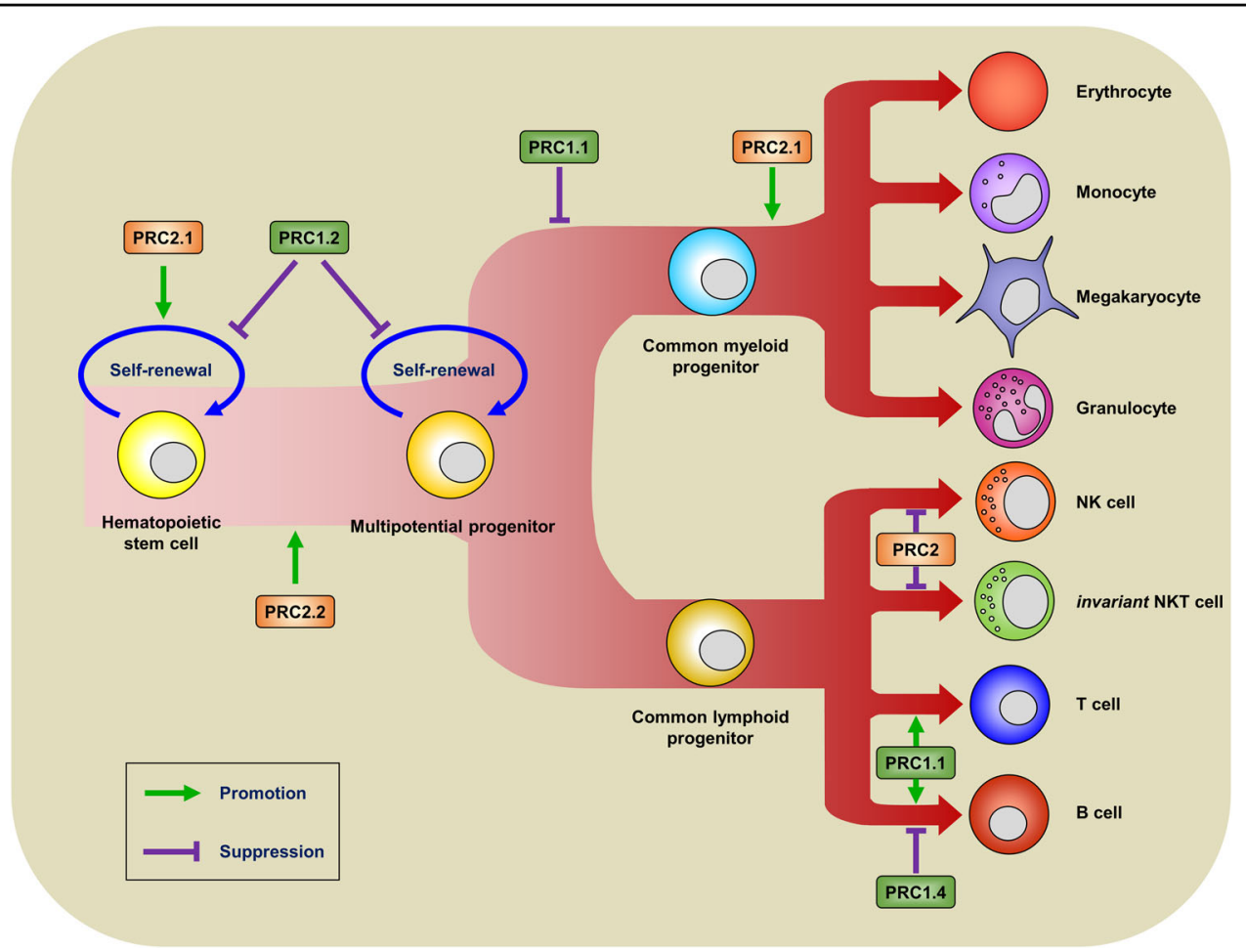

Fig. 2 Functional contribution of PcG proteins during immune cell differentiation. Schematic representation of particular PRC2 or PRC1 complexes involved in hematopoiesis according to the 'hierarchical repressive model'.

the inhibition of PcG function (http://www.ebi.ac.uk/ arrayexpress/). Most of the RNA-seq data were acquired from embryonic stem cells (ES cells), HSCs (LSK cells, LSK, $\mathrm{Lin}^{-} \mathrm{Sca}-1^{+} \mathrm{c}-\mathrm{kit}^{+}$cells), hematopoietic stem and progenitor cells (HSPC), and cancer cell lines including leukemia, multiple myeloma, sarcoma, ovarian tumor, and gastric cancer cell lines because the initial identification of PcG function emphasized the maintenance of self-renewal activity (Table 4). To expand the collection of differentially expressed gene (DEG) data, RNA-seq analyses need to be performed using a broad range of immune cells, including B cells, monocytes, dendritic cells, mast cells, and polymorphonuclear cells. All DEGs identified in PcGdefective cells might not be direct targets of PcG proteins. Chip-seq data might be needed to verify whether these DEGs are direct targets of PcG proteins. Table 5 summarizes the current ChIP-seq databases for specific cell types (http://www.ebi.ac.uk/arrayexpress/). The DNAbinding sites of most core subunits of PRC2, except for RBBP, and the core subunits of PRC1.2 (RING1B and MEL18) have been analyzed by ChIP-seq (Table 5). The DNA-binding sites of some paralogs of CBX and Jarid2, a subunit of PRC2.2, have also been analyzed (Table 5). However, most of the ChIP-seq data were acquired from stem cell lineages with few exceptions (Table 5). Therefore, a broad range of cells need to be analyzed by Chip- seq using antibodies against the remainder of the PcG proteins, including RBBP, BMI-1, and PHC, to identify novel target genes repressed by PcG proteins.

\section{Therapeutic agents for treating hematopoietic malignancies by inhibiting the activity of PcG proteins}

Since the function of PcG proteins is important to maintain the self-renewal activity of stem cells, PcG proteins might act as oncogenes to facilitate tumorigenesis. In support of this idea, high expression of EZH2 has been observed in several hematopoietic malignancies, including myelodysplastic syndromes, acute myeloid leukemia, and various types of lymphomas ${ }^{87-89}$. In particular, EZH2 deficiency in mice can inhibit leukemogenesis by decreasing the proliferation rate of leukemia ${ }^{90}$. Consistent with these observations, the expression levels of canonical subunits of PRC1, including BMI1, CBX7, CBX8, and RING1A, are elevated in many hematopoietic-originating tumors ${ }^{88,91,92}$. A mouse model involving Bmil-deficient mice with transformed cells also supports the notion that BMI1 can act as an oncogene in some hematopoietic malignant cells ${ }^{93}$. However, the loss of function of PcG proteins by mutation or deletion might also cause hematopoietic malignancies ${ }^{91}$. In particular, defects in core subunits of PRC2, including EZH2, EED, and SUZ12, have been found in various acute lymphoblastic 
Table 4 RNA-seq or gene-chip data from loss or gain of function of each PcG subunit protein ${ }^{a, b}$.

\begin{tabular}{|c|c|c|c|}
\hline Unit & Data ID & Target cell (cell line) & Functional alteration (method) \\
\hline \multirow[t]{2}{*}{$\mathrm{EZH} 1$} & E-GEOD-36288 & LSK $\left(\right.$ Lin $^{-}$Sca- $\left.1^{+}{ }^{-}-\mathrm{kit}^{+}\right)$cells & Loss of function $(\mathrm{KO})$ \\
\hline & E-GEOD-59090 & $\mathrm{CD}_{4} 4^{+}$hematopoietic stem and progenitor cell (HSPC)-derived proerythroblasts & Loss of function (shRNA) \\
\hline $\mathrm{EZH} 1 / \mathrm{EZH} 2$ & E-GEOD-62198 & MLL-AF9 induced leukemia progenitor & Loss of function (inhibitor, UNC1999) \\
\hline \multirow[t]{11}{*}{$\mathrm{EZH} 2$} & E-GEOD-59090 & $\mathrm{CD}_{4} 4^{+}$HSPC-derived proerythroblasts & Loss of function (shRNA) \\
\hline & E-GEOD-71870 & Ovarian tumor (OC8 cell line) & Loss of function (shRNA) \\
\hline & E-GEOD-71671 & Monocyte (THP-1 cell line) & Loss of function (inhibitor, GSK126) \\
\hline & E-MTAB-3227 & Gastric cancer (MKN45 cell line) & Loss of function (siRNA) \\
\hline & E-GEOD-82072 & Megakaryocyte-erythrocyte precursor & Loss of function (cKO; scl-cre) \\
\hline & E-GEOD-82073 & Long-term-hematopoietic stem cell (LT-HSC) & Loss of function (cKO; scl-cre) \\
\hline & E-MTAB-2893 & Chronic myeloid leukemia $\mathrm{CD}_{3} 4^{+}$cells & Loss of function (inhibitor, GSK343) \\
\hline & E-MTAB-3552 & Nonchronic myeloid leukemia $\mathrm{CD}_{3} 4^{+}$cells & Loss of function (inhibitor, GSK343) \\
\hline & E-MTAB-5766 & Acute myeloid leukemia cell (F-36P, MOLM-13, and OCI-M2 cell line) & Loss of function (shRNA) \\
\hline & E-MTAB-7739 & Macroglobulinemia cell (RPCI-WM1 cell line) & Loss of function (inhibitor, Tazemetostat) \\
\hline & GSE101316 & Mouse bone marrow-derived macrophage & Loss of function (KO) \\
\hline \multirow[t]{6}{*}{ EED } & E-GEOD-12982 & Embryonic stem cell (ES cell) & Loss of function (KO) \\
\hline & E-GEOD-49305 & ES cell & Loss of function $(\mathrm{KO})$ \\
\hline & E-GEOD-62198 & MLL-AF9 induced leukemia progenitor & Loss of function (shRNA) \\
\hline & E-GEOD-53508 & ES cell & Loss of function $(\mathrm{KO})$ \\
\hline & E-GEOD-53506 & ES cell & Loss of function (KO) \\
\hline & E-GEOD-59090 & $\mathrm{CD}^{+} 4^{+} \mathrm{HSPC}$-derived proerythroblasts & Loss of function (shRNA) \\
\hline \multirow[t]{4}{*}{ SUZ12 } & E-GEOD-31354 & ES cell & Loss of function (KO) \\
\hline & E-GEOD-53508 & ES cell & Loss of function (Genetrap) \\
\hline & E-GEOD-59090 & $\mathrm{CD}^{+} 4^{+} \mathrm{HSPC}$-derived proerythroblasts & Loss of function (shRNA) \\
\hline & E-GEOD-60808 & HSPC & Loss of function (shRNA) \\
\hline Jarid2 & E-GEOD-60808 & HSPC & Loss of function (shRNA) \\
\hline RING1A & E-MTAB-5661 & ES cell & Loss of function (KO) \\
\hline RING1A/B & E-MTAB-5661 & ES cell & Loss of function (KO, cKO) \\
\hline \multirow[t]{3}{*}{ RING1B } & E-GEOD-67868 & ES cell & Loss of function (KO) \\
\hline & E-GEOD-69824 & ES cell & Loss of function (KO) \\
\hline & E-GEOD-71007 & Ewing's sarcoma (A4573, A673, ES1, and TC71 cell line) & Loss of function (shRNA) \\
\hline Mel18 & E-GEOD-67868 & Embryonic stem cell & Loss of function (shRNA) \\
\hline \multirow[t]{6}{*}{ Bmi1 } & E-GEOD-21912 & Multiple myeloma(RPMI-8226 cell line) & Loss of function (shRNA) \\
\hline & E-GEOD-19796 & HSPC & Loss of function (KO) \\
\hline & E-GEOD-20958 & ES cell and HSC & Gain of function (overexpression) \\
\hline & E-GEOD-31086 & Common myeloid progenitor & Loss of function (KO) \\
\hline & E-GEOD-54262 & Erythroleukemia (K562 cell line) and Chronic myeloid leukemia & Loss of function (shRNA) \\
\hline & E-GEOD-71007 & Ewing's sarcoma (A673 and TC71 cell line) & Loss of function (shRNA) \\
\hline Cbx7 & E-GEOD-34191 & ES cell & Loss of function (shRNA) \\
\hline PCGF1 & E-GEOD-33280 & HSPC & Loss of function (shRNA) \\
\hline PCGF3/5 & E-MTAB-5642 & ES cell & Loss of function (KO) \\
\hline
\end{tabular}

KO knockout mice, $c K O$ conditional knockout mice.

anformation in Table 4 was acquired from https://www.ebi.ac.uk/arrayexpress/.

${ }^{b}$ Each reference for Table 4 is contained within contents of each Data ID.

leukemia and myelodysplastic syndromes ${ }^{94-97}$. Therefore, at least PRC2 can act as an oncogene or a tumor suppressor depending on the type of hematopoietic malignant cells involved $^{91}$. Further study is needed to define the mechanisms underlying the dual functions of these proteins in tumorigenesis.

Table 6 summarizes the inhibitors of PcG proteins applied to clinical trials in hematopoietic malignancies and other types of tumors. Major groups of inhibitors target EZH enzyme activity (Table 6). Most EZH2 inhibitors undergoing clinical trials compete with SAM for binding to the SET domain ${ }^{98}$. Among the competitive inhibitors of EZH, tazemetostat (EPZ6438), an orally administered small chemical, has been applied to a broad range of malignant cell types, including lymphoma, sarcoma, mesothelioma, ovarian cancers and advanced solid tumors (Table 6) ${ }^{98}$. Other inhibitors of PcG proteins that are currently undergoing clinical trials target EED and BMI-1 activity (Table 6). MAK683 is an allosteric EED inhibitor that 
Table 5 ChIP-seq data of each PcG subunit protein ${ }^{a, b}$.

\begin{tabular}{|c|c|c|}
\hline Unit & Data ID & Target cell (cell line) \\
\hline $\mathrm{EZH1}$ & E-GEOD-59090 & $\begin{array}{l}\mathrm{CD}^{+} 4^{+} \text {hematopoietic stem and progenitor } \\
(\mathrm{HSPC}) \text {-derived proerythroblasts }\end{array}$ \\
\hline \multirow[t]{18}{*}{$\mathrm{EZH} 2$} & E-GEOD-18776 & Mouse embryonic stem cell (ES cell) \\
\hline & E-MTAB-1305 & Human ES cell \\
\hline & E-GEOD-51079 & In vitro cultured $T h_{1}$ and $T h_{2}$ cell \\
\hline & E-GEOD-42706 & mouse resting B cell (CD43- B cell) \\
\hline & E-GEOD-49178 & ES cell \\
\hline & E-GEOD-52300 & Human liver cancer (HepG2 cell line) \\
\hline & E-GEOD-48518 & induced pluripotent stem cell \\
\hline & E-GEOD-46536 & ES cell \\
\hline & E-GEOD-53495 & $\begin{array}{l}\text { Human embryonic kidney cell (293T Rex } \\
\text { cell line) }\end{array}$ \\
\hline & E-GEOD-57632 & Multiple myeloma \\
\hline & E-GEOD-61586 & Neural stem cell and glioma (SF7761 cell line) \\
\hline & E-MTAB-2002 & Mouse ES cell \\
\hline & E-GEOD-47082 & Mouse ES cell \\
\hline & E-GEOD-59090 & $\mathrm{CD}_{4} 4^{+} \mathrm{HSPC}$-derived proerythroblasts \\
\hline & E-GEOD-70440 & Mammary gland \\
\hline & E-GEOD-60160 & Mouse ES cell \\
\hline & E-MTAB-6410 & Chronic lymphocytic leukemia \\
\hline & GSE101320 & Mouse bone marrow-derived macrophages \\
\hline \multirow[t]{3}{*}{ EED } & E-GEOD-61902 & Spermatocyte \\
\hline & E-GEOD-59090 & $\mathrm{CD} 4^{+} \mathrm{HSPC}$-derived proerythroblasts \\
\hline & E-MTAB-6165 & Mouse ES cell \\
\hline \multirow[t]{14}{*}{ SUZ12 } & E-GEOD-34483 & Mouse ES cell \\
\hline & E-GEOD-42616 & Mouse ES cell \\
\hline & E-GEOD-44286 & Mouse ES cell \\
\hline & E-GEOD-52300 & Human liver cancer (HepG2 cell line) \\
\hline & E-MTAB-2481 & Mouse ES cell \\
\hline & E-GEOD-55698 & ES cell \\
\hline & E-GEOD-52619 & Mouse ES cell \\
\hline & E-GEOD-47528 & Primary $\mathrm{CD}^{+}{ }^{+}$helper $\mathrm{T}$ cell \\
\hline & E-GEOD-58023 & ES cell \\
\hline & E-GEOD-62437 & MLL-AF9 transformed leukemia \\
\hline & E-GEOD-43915 & Mouse E13.5 brain \\
\hline & E-GEOD-59090 & $\mathrm{CD}_{4} 4^{+} \mathrm{HSPC}$-derived proerythroblasts \\
\hline & E-GEOD-57926 & Mouse heart and embryonic fibroblast cell \\
\hline & E-GEOD-61148 & $\begin{array}{l}\text { Mouse thymocyte and thymic T cell tumor } \\
\text { (ILC87 cell line) }\end{array}$ \\
\hline
\end{tabular}

Table 5 continued

\begin{tabular}{|c|c|c|}
\hline Unit & Data ID & Target cell (cell line) \\
\hline & E-GEOD-74330 & Mouse ES cell \\
\hline & E-GEOD-83082 & Mouse ES cell \\
\hline Jarid2 & E-GEOD-19708 & Mouse ES cell \\
\hline \multirow[t]{6}{*}{ RING1B } & E-GEOD-23716 & Mouse ES cell \\
\hline & E-GEOD-55698 & ES cell \\
\hline & E-GEOD-43915 & Mouse E13.5 brain \\
\hline & E-GEOD-67868 & Mouse ES cell \\
\hline & E-GEOD-72164 & Mouse ES cell \\
\hline & E-GEOD-74330 & Mouse ES cell \\
\hline \multirow[t]{2}{*}{ MEL18 } & E-GEOD-67868 & Mouse ES cell \\
\hline & E-GEOD-74330 & Mouse ES cell \\
\hline CBX2 & E-GEOD-29611 & Erythroleukemia (K562 cell line) \\
\hline \multirow[t]{4}{*}{ CBX3 } & E-GEOD-29611 & Erythroleukemia (K562 cell line) \\
\hline & E-GEOD-32465 & $\begin{array}{l}\text { Human colon cancer (HCT116 cell line) and } \\
\text { erythroleukemia (K562 cell line) }\end{array}$ \\
\hline & E-GEOD-28115 & Human colon cancer (HCT116 cell line) \\
\hline & E-GEOD-44242 & $\begin{array}{l}\text { Mouse ES cell and induced pluripotent } \\
\text { stem cell }\end{array}$ \\
\hline \multirow[t]{3}{*}{ CBX7 } & E-GEOD-23716 & Mouse ES cell \\
\hline & E-GEOD-42466 & Mouse ES cell \\
\hline & E-GEOD-42706 & mouse resting B cell (CD43- B cell) \\
\hline \multirow[t]{2}{*}{ CBX8 } & E-GEOD-29611 & Erythroleukemia (K562 cell line) \\
\hline & E-GEOD-54052 & Mouse ES cell \\
\hline
\end{tabular}

anformation in Table 5 was acquired from https://www.ebi.ac.uk/arrayexpress/. ${ }^{b}$ Each reference for Table 5 is contained within contents of each Data ID.

drives conformational changes in the H3K27me3-binding pocket of EED upon binding ${ }^{99}$. These conformational changes in EED further prevent the interaction between EED and EZH2, thus blocking H3K27me3 ${ }^{99}$. PTC596 is a BMI-1 inhibitor that can facilitate the degradation of BMI- 1 by inducing the cyclin-dependent kinase 1-mediated biphosphorylation of the $N$-terminus of BMI- $1^{100}$.

\section{Conclusion and future prospects}

In this review, we highlighted the structural diversity of mammalian PRC2 and PRC1 complexes related to their functional contribution to immune regulation. We also described currently available RNA-seq and ChIP-seq data that could be used to mine new target loci of PcG proteins. Finally, we listed the PcG inhibitors currently undergoing clinical trials. Many previous reports have demonstrated that PcG proteins are major chromatin modifiers that can modulate many biological processes by influencing specific gene repression, mainly using loss-of-function models. 


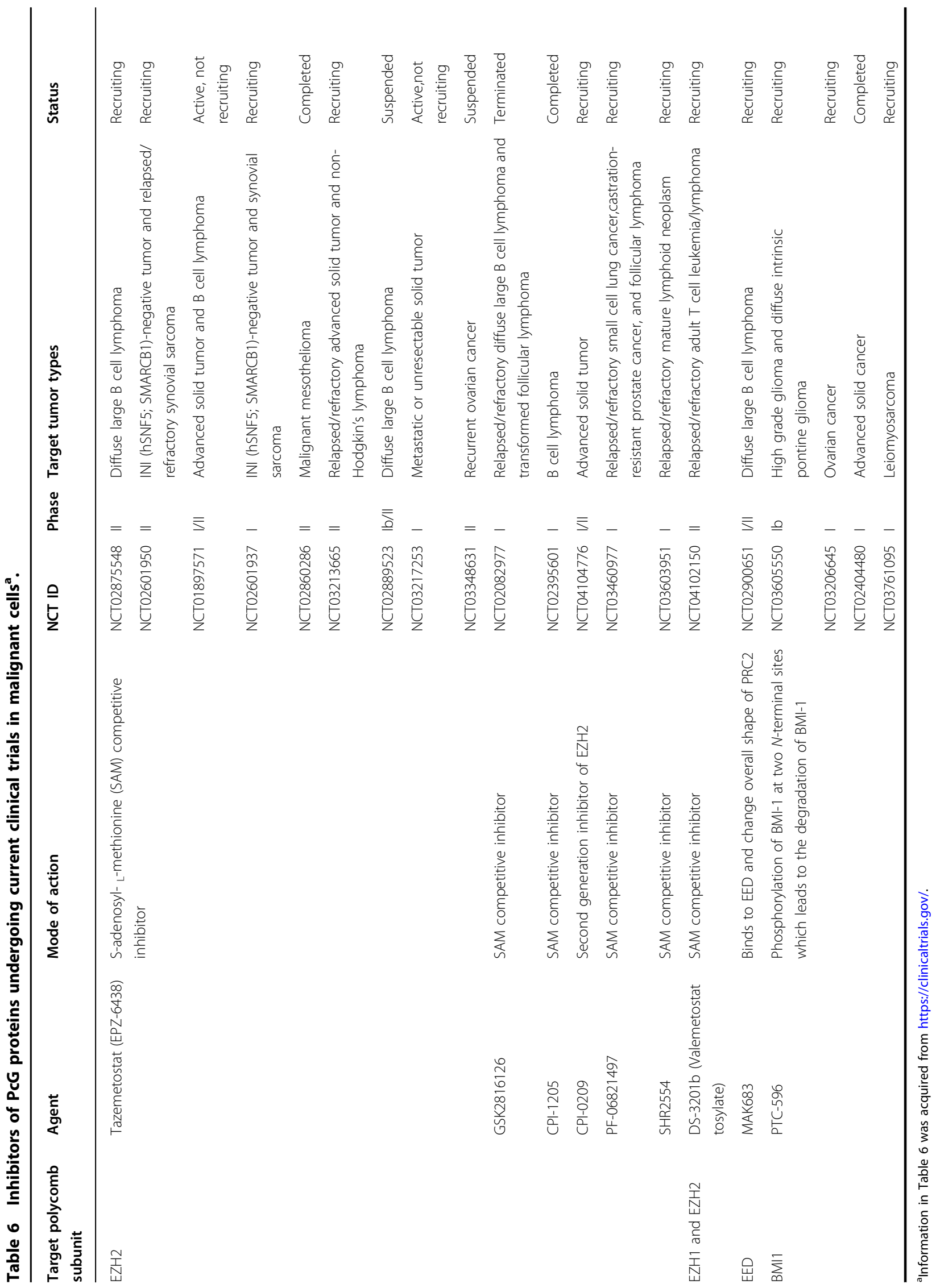


Unfortunately, we still do not know how many different types of PRCs exist in nature due to structural heterogeneity caused by many paralogs and accessory proteins recruited by PRC complexes. We also do not know how each different PRC containing a particular paralog as a subunit contributes to the phenotype of a specific cell type. Solving these unknown issues might provide novel targets for PcGmediated gene regulation and expand the range of PcG proteins considered as therapeutic targets to treat other human diseases in addition to cancer.

\section{Acknowledgements}

This work was supported by a National Research Foundation of Korea (NRF) grant funded by the Korean government (MSIT) (2019R1A2C2088046) and an intramural research program funded by a Korea University grant (2017)

\section{Conflict of interest}

The authors declare that they have no conflict of interest.

\section{Publisher's note}

Springer Nature remains neutral with regard to jurisdictional claims in published maps and institutional affiliations.

Received: 3 January 2020 Revised: 21 April 2020 Accepted: 25 May 2020. Published online: 7 July 2020

\section{References}

1. Bannister, A. J. \& Kouzarides, T. Regulation of chromatin by histone modifications. Cell Res. 21, 381-395 (2011).

2. Greer, E. L. \& Shi, Y. Histone methylation: a dynamic mark in health, disease and inheritance. Nat. Rev. Genet. 13, 343-357 (2012).

3. Sparmann, A. \& van Lohuizen, M. Polycomb silencers control cell fate, development and cancer. Nat. Rev. Cancer 6, 846-856 (2006).

4. Aranda, S., Mas, G. \& Di Croce, L. Regulation of gene transcription by polycomb proteins. Sci. Adv. 1, e1500737 (2015).

5. Schwartz, Y. B. \& Pirrotta, V. Ruled by ubiquitylation: a new order for polycomb recruitment. Cell Rep. 8, 321-325 (2014).

6. Schuettengruber, B. \& Cavalli, G. Recruitment of polycomb group complexes and their role in the dynamic regulation of cell fate choice. Development 136 3531-3542 (2009).

7. Connelly, K. E. \& Dykhuizen, E. C. Compositional and functional diversity of canonical PRC1 complexes in mammals. Biochim. Biophys. Acta Gene Regul. Mech. 1860, 233-245 (2017).

8. Oktaba, K. et al. Dynamic regulation by polycomb group protein complexes controls pattern formation and the cell cycle in Drosophila. Dev. Cell 15, 877-889 (2008).

9. Margueron, R. et al. Role of the polycomb protein EED in the propagation of repressive histone marks. Nature 461, 762-767 (2009),

10. Chittock, E. C., Latwiel, S., Miller, T. C. \& Müller, C. W. Molecular architecture of polycomb repressive complexes. Biochem. Soc. Trans. 45, 193-205 (2017).

11. Laugesen, A., Højfeldt, J. W. \& Helin, K. Molecular mechanisms directing PRC2 recruitment and H3K27 methylation. Mol. Cell 74, 8-18 (2019).

12. Di Croce, L. \& Helin, K. Transcriptional regulation by polycomb group proteins. Nat. Struct. Mol. Biol. 20, 1147-1155 (2013).

13. Vidal, M. \& Starowicz, K. Polycomb complexes PRC1 and their function in hematopoiesis. Exp. Hematol. 48, 12-31 (2017).

14. Ferrari, K. J. et al. Polycomb-dependent H3K27me1 and H3K27me2 regulate active transcription and enhancer fidelity. Mol. Cell 53, 49-62 (2014).

15. Sneeringer, C. J. et al. Coordinated activities of wild-type plus mutant EZH2 drive tumor-associated hypertrimethylation of lysine 27 on histone $\mathrm{H} 3$ (H3K27) in human B-cell lymphomas. Proc. Natl Acad. Sci. USA 107, 20980-20985 (2010).

16. Zee, B. M., Britton, L.-M. P., Wolle, D., Haberman, D. M. \& Garcia, B. A. Origins and formation of histone methylation across the human cell cycle. Mol. Cell. Biol. 32, 2503-2514 (2012).
17. Cao, R. et al. Role of histone $\mathrm{H} 3$ lysine 27 methylation in polycomb-group silencing. Science 298, 1039-1043 (2002).

18. Cao, R. \& Zhang, Y. SUZ12 is required for both the histone methyltransferase activity and the silencing function of the EED-EZH2 complex. Mol. Cell $\mathbf{1 5}$ 57-67 (2004).

19. Nekrasov, M., Wild, B. \& Müller, J. Nucleosome binding and histone methyltransferase activity of Drosophila PRC2. EMBO Rep. 6, 348-353 (2005).

20. Gao, Z. et al. PCGF homologs, CBX proteins, and RYBP define functionally distinct PRC1 family complexes. Mol. Cell 45, 344-356 (2012).

21. Junco, S. E. et al. Structure of the polycomb group protein PCGF1 in complex with BCOR reveals basis for binding selectivity of PCGF homologs. Structure 21, 665-671 (2013).

22. Rinn, J. L. et al. Functional demarcation of active and silent chromatin domains in human HOX loci by noncoding RNAs. Cell 129, 1311-1323 (2007).

23. $\mathrm{Xu}, \mathrm{C} . \& \mathrm{Min}, \mathrm{J}$. Structure and function of WD40 domain proteins. Protein Cell 2, 202-214 (2011).

24. Kuzmichev, A., Nishioka, K, Erdjument-Bromage, H., Tempst, P. \& Reinberg, D. Histone methyltransferase activity associated with a human multiprotein complex containing the Enhancer of Zeste protein. Genes Dev. 16, 2893-2905 (2002)

25. Davidovich, C. \& Cech, T. R. The recruitment of chromatin modifiers by long noncoding RNAs: lessons from PRC2. RNA 21, 2007-2022 (2015).

26. Laugesen, A. \& Helin, K. Chromatin repressive complexes in stem cells, development, and cancer. Cell Stem Cell 14, 735-751 (2014).

27. Hauri, S. et al. A high-density map for navigating the human polycomb complexome. Cell Rep. 17, 583-595 (2016).

28. Alekseyenko, A. A., Gorchakov, A. A., Kharchenko, P. V. \& Kuroda, M. I. Reciprocal interactions of human C10orf12 and C17orf96 with PRC2 revealed by BioTAP-XL cross-linking and affinity purification. Proc. Natl Acad. Sci. USA 111, 2488-2493 (2014)

29. Kloet, S. L. et al. The dynamic interactome and genomic targets of polycomb complexes during stem-cell differentiation. Nat. Struct. Mol. Biol. 23, 682-690 (2016).

30. Boulay, G., Rosnoblet, C., Guérardel, C., Angrand, P.-O. \& Leprince, D. Functional characterization of hPCL3 (human Polycomb-like 3) isoforms identifies them as components of distinct EZH2 protein complexes. Biochem. J. 434, 333-342 (2011).

31. Ballaré, $\mathrm{C}$. et al. Phf19 links methylated Lys36 of histone $\mathrm{H} 3$ to regulation of polycomb activity. Nat. Struct. Mol. Biol. 19, 1257-1265 (2012).

32. Li, H. et al. Polycomb-like proteins link the PRC2 complex to CpG islands. Nature 549, 287-291 (2017).

33. Perino, M. et al. MTF2 recruits polycomb repressive complex 2 by helicalshape-selective DNA binding. Nat. Genet. 50, 1002-1010 (2018).

34. Conway, E. et al. A family of vertebrate-specific polycombs encoded by the LCOR/LCORL genes balance PRC2 subtype activities. Mol. Cell 70, 408-421 (2018).

35. Beringer, $M$. et al. EPOP functionally links elongin and polycomb in pluripotent stem cells. Mol. Cell 64, 645-658 (2016).

36. Kasinath, $V$. et al. Structures of human PRC2 with its cofactors AEBP2 and JARID2. Science 359, 940-944 (2018).

37. Cooper, S. et al. Jarid2 binds mono-ubiquitylated H2A lysine 119 to mediate crosstalk between polycomb complexes PRC1 and PRC2. Nat. Commun. 7 13661 (2016).

38. van den Boom, V. et al. Non-canonical PRC1. 1 targets active genes independent of H3K27me3 and is essential for leukemogenesis. Cell Rep. 14 332-346 (2016).

39. de Napoles, M. et al. Polycomb group proteins Ring1A/B link ubiquitylation of histone $\mathrm{H} 2 \mathrm{~A}$ to heritable gene silencing and X inactivation. Dev. Cell 7, 663-676 (2004).

40. Wang, $\mathrm{H}$. et al. Role of histone $\mathrm{H} 2 \mathrm{~A}$ ubiquitination in polycomb silencing. Nature 431, 873-878 (2004).

41. Srivastava, A., McGrath, B. \& Bielas, S. L. Histone H2A monoubiquitination in neurodevelopmental disorders. Trends Genet. 33, 566-578 (2017)

42. Fischle, W. et al. Molecular basis for the discrimination of repressive methyllysine marks in histone $\mathrm{H} 3$ by polycomb and HP1 chromodomains. Genes Dev. 17, 1870-1881 (2003).

43. Kaustov, L. et al. Recognition and specificity determinants of the human cbx chromodomains. J. Biol. Chem. 286, 521-529 (2011).

44. Robinson, A. K. et al. The growth-suppressive function of the polycomb group protein polyhomeotic is mediated by polymerization of its sterile alpha motif (SAM) domain. J. Biol. Chem. 287, 8702-8713 (2012).

45. Kim, C. A. \& Francis, N. J. Chromatin: polycomb group SAMs unite. Curr. Biol. 26, R710-R712 (2016). 
46. Levine, S. S. et al. The core of the polycomb repressive complex is compositionally and functionally conserved in flies and humans. Mol. Cell. Biol. 22 6070-6078 (2002).

47. Bezsonova, I. et al. Ring1B contains a ubiquitin-like docking module for interaction with Cbx proteins. Biochemistry 48, 10542-10548 (2009).

48. Wang, R. et al. Polycomb group targeting through different binding partners of RING1B C-terminal domain. Structure 18, 966-975 (2010).

49. Taherbhoy, A. M., Huang, O. W. \& Cochran, A. G. BMI1-RING1B is an autoinhibited RING E3 ubiquitin ligase. Nat. Commun. 6, 7621 (2015).

50. Farcas, A. M. et al. KDM2B links the polycomb repressive complex 1 (PRC1) to recognition of CpG islands. Elife 1, e00205 (2012).

51. Blackledge, N. P. et al. Variant PRC1 complex-dependent H2A ubiquitylation drives PRC2 recruitment and polycomb domain formation. Cell 157 1445-1459 (2014).

52. Mendenhall, E. M. et al. GC-rich sequence elements recruit PRC2 in mammalian ES cells. PLoS Genet. 6, e1001244 (2010).

53. Brown, J. L., Mucci, D., Whiteley, M., Dirksen, M.-L. \& Kassis, J. A. The Drosophila polycomb group gene pleiohomeotic encodes a DNA binding protein with homology to the transcription factor YY1. Mol. Cell 1, 1057-1064 (1998).

54. Atchison, L., Ghias, A., Wilkinson, F., Bonini, N. \& Atchison, M. L. Transcription factor YY1 functions as a PCG protein in vivo. EMBO J. 22, 1347-1358 (2003).

55. Basu, A., Wilkinson, F. H., Colavita, K., Fennelly, C. \& Atchison, M. L. YY1 DNA binding and interaction with YAF2 is essential for polycomb recruitment. Nucleic Acids Res. 42, 2208-2223 (2013).

56. Hidalgo, I. et al. Ezh1 is required for hematopoietic stem cell maintenance and prevents senescence-like cell cycle arrest. Cell Stem Cell 11, 649-662 (2012).

57. O'Carroll, D. et al. The polycomb-group Gene Ezh2 is required for early mouse development. Mol. Cell. Biol. 21, 4330-4336 (2001).

58. Mochizuki-Kashio, M. et al. Dependency on the polycomb gene Ezh2 distinguishes fetal from adult hematopoietic stem cells. Blood 118, 6553-6561 (2011).

59. Tumes, D. J. et al. The polycomb protein Ezh2 regulates differentiation and plasticity of CD4+ T helper type 1 and type 2 cells. Immunity $\mathbf{3 9}$, 819-832 (2013)

60. Zhang, Y. et al. The polycomb repressive complex 2 governs life and death of peripheral T cells. Blood 124, 737-749 (2014).

61. Gray, S. M., Amezquita, R. A., Guan, T., Kleinstein, S. H. \& Kaech, S. M. Polycomb repressive complex 2-mediated chromatin repression guides effector CD8+ T cell terminal differentiation and loss of multipotency. Immunity 46, 596-608 (2017).

62. Yin, J. et al. Ezh2 regulates differentiation and function of natural killer cells through histone methyltransferase activity. Proc. Natl Acad. Sci. USA 112 15988-15993 (2015).

63. Tumes, D. et al. Ezh2 controls development of natural killer T cells, which cause spontaneous asthma-like pathology. J. Allergy Clin. Immunol. 144, 549-560 (2019).

64. Xie, H. et al. Polycomb repressive complex 2 regulates normal hematopoietic stem cell function in a developmental-stage-specific manner. Cell Stem Cell 14, 68-80 (2014).

65. Simon, C. et al. A key role for EZH2 and associated genes in mouse and human adult T-cell acute leukemia. Genes Dev. 26, 651-656 (2012).

66. Lee, S. C. et al. Polycomb repressive complex 2 component Suz12 is required for hematopoietic stem cell function and lymphopoiesis. Blood 126, 167-175 (2015).

67. Lessard, J. \& Sauvageau, G. Bmi-1 determines the proliferative capacity of normal and leukaemic stem cells. Nature 423, 255-260 (2003).

68. Park, I.-k et al. Bmi-1 is required for maintenance of adult self-renewing haematopoietic stem cells. Nature 423, 302-305 (2003).

69. Yamashita, M. et al. Bmi1 regulates memory CD4 T cell survival via repression of the Noxa gene. J. Exp. Med. 205, 1109-1120 (2008).

70. Miyazaki, M. et al. Thymocyte proliferation induced by pre-T cell receptor signaling is maintained through polycomb gene product Bmi-1-mediated Cdkn2a repression. Immunity 28, 231-245 (2008).

71. Hosokawa, H. et al. Regulation of Th2 cell development by polycomb group gene bmi-1 through the stabilization of GATA3. J. Immunol. 177, 7656-7664 (2006)

72. Iwama, A. et al. Enhanced self-renewal of hematopoietic stem cells mediated by the polycomb gene product Bmi-1. Immunity 21, 843-851 (2004).

73. Kimura, M. et al. Regulation of Th2 cell differentiation by mel-18, a mammalian polycomb group gene. Immunity 15, 275-287 (2001).
74. Ohta, $\mathrm{H}$. et al. Polycomb group gene rae28 is required for sustaining activity of hematopoietic stem cells. J. Exp. Med. 195, 759-770 (2002).

75. Kim, J. Y. et al. Defective long-term repopulating ability in hematopoietic stem cells lacking the polycomb-group gene rae28. Eur. J. Haematol. 73, 75-84 (2004).

76. Bae, J. et al. Phc2 controls hematopoietic stem and progenitor cell mobilization from bone marrow by repressing Vcam1 expression. Nat. Commun. 10, 3496 (2019).

77. Coré, N. et al. Altered cellular proliferation and mesoderm patterning in polycomb-M33-deficient mice. Development 124, 721-729 (1997).

78. Liu, B. et al. Cbx4 regulates the proliferation of thymic epithelial cells and thymus function. Development 140, 780-788 (2013).

79. Tan, J. et al. CBX8, a polycomb group protein, is essential for MLL-AF9induced leukemogenesis. Cancer Cell 20, 563-575 (2011).

80. Béquelin, W. et al. EZH2 and BCL6 cooperate to assemble CBX8-BCOR complex to repress bivalent promoters, mediate germinal center formation and lymphomagenesis. Cancer Cell 30, 197-213 (2016).

81. Calés, C. et al. Inactivation of the polycomb group protein Ring1B unveils an antiproliferative role in hematopoietic cell expansion and cooperation with tumorigenesis associated with Ink4a deletion. Mol. Cell. Biol. 28, 1018-1028 (2008).

82. Ikawa, T. et al. Conversion of T cells to B cells by inactivation of polycombmediated epigenetic suppression of the B-lineage program. Genes Dev. 30 2475-2485 (2016).

83. Shen, $\mathrm{X}$. et al. EZH1 mediates methylation on histone $\mathrm{H} 3$ lysine 27 and complements $\mathrm{EZH} 2$ in maintaining stem cell identity and executing pluripotency. Mol. Cell 32, 491-502 (2008).

84. Ezhkova, E. et al. EZH1 and EZH2 cogovern histone H3K27 trimethylation and are essential for hair follicle homeostasis and wound repair. Genes Dev. $\mathbf{2 5}$ 485-498 (2011).

85. Jacobs, J. J., Kieboom, K., Marino, S., DePinho, R. A. \& van Lohuizen, M. The oncogene and polycomb-group gene bmi-1 regulates cell proliferation and senescence through the ink4a locus. Nature 397, 164-168 (1999).

86. Lessard, J., Baban, S. \& Sauvageau, G. Stage-specific expression of polycomb group genes in human bone marrow cells. Blood 91, 1216-1224 (1998).

87. Lund, K. Adams, P. D. \& Copland, M. EZH2 in normal and malignant hematopoiesis. Leukemia 28, 44-49 (2014).

88. $\mathrm{Xu}, \mathrm{F}$. et al. Overexpression of the EZH2, RING1 and BMl1 genes is common in myelodysplastic syndromes: relation to adverse epigenetic alteration and poor prognostic scoring. Ann. Hematol. 90, 643-653 (2011).

89. Raaphorst, F. M. et al. Coexpression of BMl-1 and EZH2 polycomb group genes in Reed-Sternberg cells of Hodgkin's disease. Am. J. Pathol. 157, 709-715 (2000).

90. Tanaka, S. et al. Ezh2 augments leukemogenicity by reinforcing differentiation blockage in acute myeloid leukemia. Blood 120, 1107-1117 (2012).

91. Takamatsu-Ichihara, E. \& Kitabayashi, I. The roles of Polycomb group proteins in hematopoietic stem cells and hematological malignancies. Int. J. Hematol. 103, 634-642 (2016).

92. Mihara, K. et al. Bmi-1 is useful as a novel molecular marker for predicting progression of myelodysplastic syndrome and patient prognosis. Blood 107, 305-308 (2006).

93. Yuan, J. et al. Bmi1 is essential for leukemic reprogramming of myeloid progenitor cells. Leukemia 25, 1335-1343 (2011).

94. Ntziachristos, P. et al. Genetic inactivation of the polycomb repressive complex 2 in T cell acute lymphoblastic leukemia. Nat. Med. 18, 298-301 (2012).

95. Nikoloski, G. et al. Somatic mutations of the histone methyltransferase gene EZH2 in myelodysplastic syndromes. Nat. Genet. 42, 665-667 (2010).

96. Ernst, $\mathrm{T}$. et al. Inactivating mutations of the histone methyltransferase gene EZH2 in myeloid disorders. Nat. Genet. 42, 722-726 (2010).

97. Score, J. et al. Inactivation of polycomb repressive complex 2 components in myeloproliferative and myelodysplastic/myeloproliferative neoplasms. Blood 119, 1208-1213 (2012)

98. Stazi, G., Zwergel, C., Mai, A. \& Valente, S. EZH2 inhibitors: a patent review (2014-2016). Expert Opin. Ther. Pat. 27, 797-813 (2017).

99. Qi, W. et al. An allosteric PRC2 inhibitor targeting the H3K27me3 binding pocket of EED. Nat. Chem. Biol. 13, 381-388 (2017).

100. Nishida, Y. et al. The novel BMl-1 inhibitor PTC596 downregulates MCL-1 and induces p53-independent mitochondrial apoptosis in acute myeloid leukemia progenitor cells. Blood Cancer J. 7, e527 (2017) 